\title{
A nonsense mutation is responsible for the RNA-negative phenotype in human citrullinaemia
}

\author{
Chi-Ming Li ${ }^{1,4}$, Hung-Kun Chao ${ }^{3}$, Yu-Fen $\mathrm{Liu}^{3,5}$ and Tsung-Sheng $\mathrm{Su}^{\star, 1,2,3}$ \\ ${ }^{1}$ Institute of Genetics, National Yang-Ming University, Taiwan, Republic of China; ${ }^{2}$ Institute of Genetics, \\ Microbiology \& Immunology, National Yang-Ming University, Taiwan, Republic of China; ${ }^{3}$ Department of Medical \\ Research and Education, Veterans General Hospital-Taipei, Taiwan, Republic of China
}

Citrullinaemia is an inborn error of metabolism resulting from a deficiency of argininosuccinate synthetase. Previous studies of RNA of argininosuccinate synthetase of citrullinaemia patients using S1 nuclease analysis have identified a class of so-called RNA-negative alleles in which no stable mRNA can be detected. To investigate the nature of mutation responsible for such a phenotype, a compound heterozygous citrullinaemia carrying an RNA-negative allele and an allele with a $3^{\prime}$ splice site mutation in intron 6 (IVS6-2A $>G$ ) was analysed. Using sequences of a DNA polymorphism and the IVS6-2A $>$ G mutation as markers, approximately equal amounts of pre-mRNAs from allelic genes were detected suggesting that RNA-negative phenotype could not be the result of defect in transcription initiation. A C-to-T transition converting the CGA arginine codon at residue 279 to a TGA termination codon (R279X) was identified by CDNA sequencing. No accumulation of partially spliced pre-mRNAs containing introns immediately upstream and downstream of the nonsense mutation was observed. In addition, no mRNA species of abnormal size was detected when cDNA from the RNA-negative allele was analysed. Hence, there is no indication of nonsense-associated altered splicing (NAS). The most likely event responsible for the RNA-negative phenotype appears to be nonsense-mediated mRNA decay (NMD). European Journal of Human Genetics (2001) 9, 685-689.

Keywords: argininosuccinate synthetase; citrullinaemia; RNA-negative phenotype; nonsense-mediated mRNA decay; splice site mutation

\section{Introduction}

Argininosuccinate synthetase (ASS) catalyses the conversion of citrulline and aspartate to argininosuccinate. The enzyme is present ubiquitously in tissues. The highest enzymatic activity is found in the liver where the enzyme acts in the urea cycle to eliminate ammonia. Deficiency of argininosuccinate synthetase results in an autosomal recessive

\footnotetext{
*Correspondence: T-S Su, Department of Medical Research and Education, Veterans General Hospital-Taipei, Taipei, Taiwan, Republic of China 11217. Tel: 886-2-2871-2121 ext. 3361; Fax: 886-2-2875-1562; E-mail: tssu@vghtpe.gov.tw

Current addresses: ${ }^{4}$ Institute of Cancer Genetics, Columbia University, New York, New York 10032, USA; ${ }^{5}$ Yu-Fen Liu, Institute of Genetics, National Yang-Ming University, Taipei, Taiwan, Republic of China Received 27 February 2001; revised 9 May 2001; accepted 6 June 2001
}

disorder, citrullinaemia. ${ }^{1}$ Patients with this disorder exhibit profound elevations of ammonia and citrulline in the blood. The majority of such patients are identified in the neonatal period with hyperammonaemic coma. The molecular basis of the disease is heterogeneous. ${ }^{2-6}$ S1 nuclease analysis of RNA from skin fibroblast cultures of citrullinaemia patients has revealed a class of so called RNA-negative alleles, in which the genomic DNA is intact by Southern blot analysis, but no stable mRNA is detected., ${ }^{2,3}$ So far, the molecular basis for this class of mutation has not been elucidated. To understand the nature of this defect, a study on such an allele was undertaken. The patient chosen, designated AC, inherited from her father an RNA-negative allele while from her mother an A-to-G transition at the -2 position of the 3' splice site of intron 6 (IVS6-2A $>$ G) resulting in skipping of exon $7 .^{6}$ 


\section{Materials and methods}

\section{Cell lines}

Skin fibroblasts established from the citrullinaemia patients, designated as $\mathrm{AC}, \mathrm{CB}, \mathrm{BBr}$ and $\mathrm{LP}$, were kindly provided by Drs AL Beaudet and WE O'Brian. HuH-7 is a human hepatoma cell line. ${ }^{7}$ Cells were cultured in Dulbecco's modified Eagle's medium with 10\% foetal calf serum.

\section{Molecular analysis}

High molecular weight DNA and total RNA were isolated as described by Sambrook et al. ${ }^{8}$ Polymerase chain reaction (PCR), reverse-transcription-PCR (RT-PCR) were performed using standard methods. ${ }^{9}$ Single-strand conformation polymorphism (SSCP) was performed as described. ${ }^{10}$

\section{Results}

Defect in the RNA-negative allele does not affect the level of intron 6-containing pre-mRNA

The mutation in the maternal allele is an A-to-G transition at the -2 position of the $3^{\prime}$ splice site of intron 6 (IVS6-2A $>G$ ) resulting in skipping of exon $7 .^{6}$ In addition, a cryptic $3^{\prime}$ splice site within exon 7 was found to be activated resulting in a small fraction of mRNA with a deletion of the first $45 \mathrm{nt}$ of exon 7 (data not shown). RNA-negative alleles may have resulted from a transcriptional defect producing less amount of pre-mRNA, a defect in RNA splicing/polyadenylation affecting pre-mRNA processing, or as a consequence of mRNA instability.

An initial effort was made to examine whether there was any reduction in pre-mRNA transcribed from RNAnegative allele to suggest a transcriptional defect. Allelic pre-mRNAs were distinguished based on their sequence variations including mutation at the $3^{\prime}$ splice site of intron 6 and a T/C polymorphism within intron 6 at 28 nt upstream of the $3^{\prime}$ splice site (unpublished data). By sequencing the cloned genomic DNA from the citrullinaemia patient $\mathrm{AC}$, the allele carrying the splice site mutation is shown to be the nucleotide $\mathrm{T}$ while the paternal allele with a normal splice site carries the nucleotide $\mathrm{C}$ (Figure 1A). When a $141 \mathrm{bp}$ DNA fragment encompassing exon 7 and intron 6 including this T/C polymorphism was PCR amplified from the genomic DNA of citrullinaemia AC and analysed by SSCP, allelic DNAs could be distinguished (Figure 1B, lane 1). Based on the SSCP profile of plasmid DNA of pAG141 and pGG141 which carry the respective allelic sequences, the upper and lower bands were assigned to IVS6-2A $>\mathrm{G}$ allele and RNA-negative allele, respectively (data not shown). When the pre-mRNA of citrullinaemia AC was similarly analysed, cDNAs derived from both alleles were detected (Figure $1 \mathrm{~B}$, lane 3 ). It is noted that signal intensity of premRNA from the RNA-negative allele is much weaker than the allele with the splice site mutation, while such difference is not observed in patient AC's genomic DNA
(Figure 1B, lane 1). Similar analysis was carried out on pre-mRNA from AC's mother who is a carrier with an allele harbouring the intron 6 splice site mutation and also heterozygous for the T/C polymorphism (Figure $1 \mathrm{~B}$, lane 4). The signal intensity of the pre-mRNA derived from the normal allele is also weaker, while the ratio of the allelic pre-mRNAs is similar to that in citrullinaemia AC (Figure 1B, comparing signal intensities of lanes 3 and 4). Thus, the RNA-negative allele contains the same level of primary transcript as does the wild type allele. Therefore, the observed increased level of pre-mRNA containing intron 6 from the allele carrying the splice site mutation must be the result of inefficiency in the removal of this intron. The result suggests that transcription rate is not affected by the mutation of the RNAnegative allele. Thus, the mutation responsible for the RNA-negative phenotype probably does not reside in the promoter or enhancer of the argininosuccinate synthetase gene.
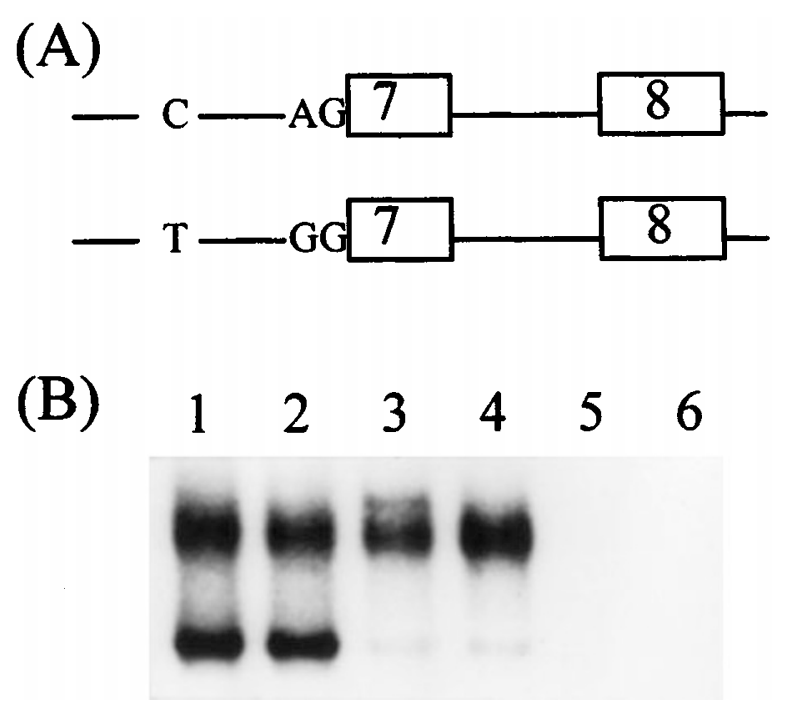

Figure 1 Analysis of pre-mRNA level containing intron 6-exon 7 by SSCP. (A) Schematic representation of a portion of the allelic argininosuccinate synthetase gene in citrullinaemia AC. (B) Comparison of pre-mRNA level among wild type allele, RNAnegative allele, and allele with mutation in the intron 6 splice site. Total RNAs of fibroblasts of citrullinaemia AC (lane 3) and AC's mother (lane 4) were reverse-transcribed using a primer that is located in exon 8 [E8, 5'- (555) GAGGTTCTCATCCATGCTCCAC (534)-3', where the first and the last residue of the primer residing within the ORF of the gene are numbered], then PCR-amplified using primers in intron $6(16,5$ '-CAGCTCTGCAGCTTACAGGC-3') and exon 7 [E7-1, 5'-(491)

GCGTACTCCATCAGGTCATTGC (470)-3'] in a reaction containing $\left[\alpha-{ }^{32} \mathrm{P}\right] \mathrm{dATP}$. Lanes 5 and 6 are controls for lanes 3 and 4, respectively, where the RT-PCR reactions were carried out in the absence of reverse transcriptase. Also amplified were genomic DNAs of citrullinaemia AC (lane 1) and AC's mother (lane 2). The amplified products were analysed by SSCP and displayed on a $6 \%$ polyacrylamide gel. 
The RNA-negative allele harbours a nonsense mutation at codon 279

For specific amplification of cDNA from the RNA-negative allele, one of the PCR primers is situated within the first $45 \mathrm{nt}$ of exon 7, the sequence of which is absent from mRNA derived from the allele harbouring the IVS6-2A $>$ G mutation. In RT-PCR reaction, a cDNA of normal size was detected (data not shown). DNA sequence analysis shows a C-to-T transition at nt 835 in this cDNA (Figure 2B). This mutation converts the CGA arginine codon at residue 279 to a TGA termination codon (R279X) (Figure 2D). The mutation is indeed present in the genomic DNA as demonstrated by direct sequencing of the PCR products generated from the genomic DNA of citrullinaemia AC (Figure 2C). The R279X mutation is within exon 12 while the normal termination codon is within the 3 '-terminal exon 16 . No other mutations were found when the entire cDNA was amplified and sequenced (data not shown). Thus, the R279X mutation is associated with the RNA-negative phenotype.

In addition to citrullinaemia AC, there are three cases of human citrullinaemia reported who carry RNA-negative alleles. ${ }^{3,5}$ A C-to-T transition at nt 835 appears in an allele of the ASS gene from citrullinaemia $C B$ while no such mutation is identified in the genomic DNAs of patients $\mathrm{BBr}$ and LP (data not shown). Therefore, the R279X mutation is also responsible for the RNA-negative phenotype in citrullinaemia $\mathrm{CB}$.

R279X mutation does not lead to splicing abnormality Studies have shown that nonsense mutations cause splicing abnormality including exon skipping, alternative splice-site choice and intron retention. ${ }^{11-13}$ Recent study of a BRCA1 mutation indicates that such splicing abnormality may in fact result from disruption of a splicing enhancer in the coding sequence. ${ }^{14}$ It is possible that the RNA-negative phenotype of the R279X mutation is in part due to disruption of the exon splicing enhancer (ESE). To examine whether premRNA containing introns adjacent to exon 12 where R279X resides are accumulated preferentially, as being observed for pre-mRNA carrying the IVS6-2A > G mutation (Figure 1), the steady-state level of pre-mRNA containing intron 11 or intron 12 was studied. The R279X mutation results in the loss of an $A v a$ I restriction site and creation of a DdeI site (Figure 3). As a result, RT-PCR product bearing R279X mutation can be distinguished from the wild type product. We found that the band intensity of the RT - PCR products from the wild type and from the mutant alleles are comparable to the PCR products derived from the genomic DNA of patient AC after AvaI or DdeI digestion. This indicates that the R279X mutation probably does not result in the retention of intron 12 (Figure $3 \mathrm{~A}$ ). To examine whether intron 11 is affected, the level of pre-mRNA bearing intron 11 was similarly analysed. Again, results reveal no accumulation of such pre-mRNA (Figure 3B). It is noteworthy that no exon skipping was detected when cDNA from the RNA-negative
(A)

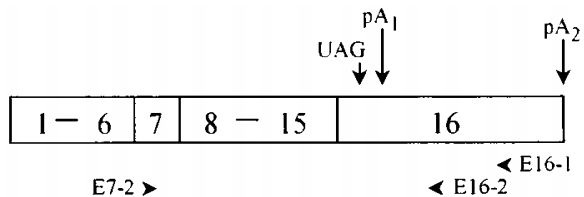

(B)

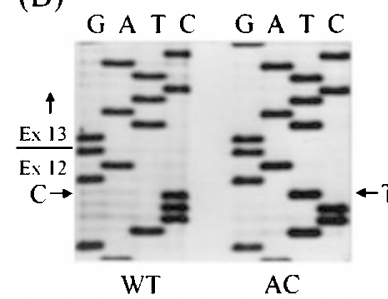

(C)
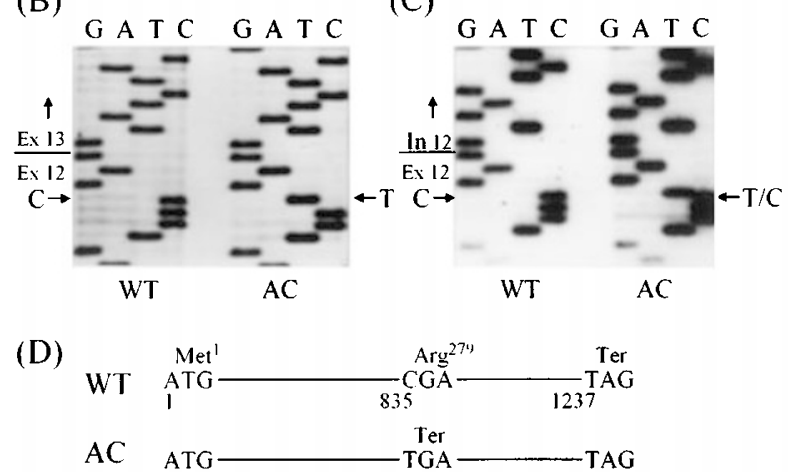

Figure 2 DNA sequence analysis of the mutation associated with the RNA negative-allele in citrullinaemia AC. (A) Schematic diagram of the mRNA structure of the human argininosuccinate synthetase gene. UAG is the authentic termination codon while $\mathrm{pA}_{1}$ and $\mathrm{pA}_{2}$ are the poly $(\mathrm{A})$ signals for the $1.7-\mathrm{kb}$ and $2.7-\mathrm{kb}$ mRNAs, respectively. The locations of primer E7-2 [5'-(445) GAATTCTACAACCGGTTCAAG (465)-3'], primer E16-1 [5'(1663) ATTCCTAGTTITTGCTGGAG (1644)-3'] and primer E162 [5'-(1598) TTCGGCAGCACTTAGGTC (1581)-3'] for RT - PCR are indicated. The drawing is not to scale. (B) DNA sequence analysis of the control plasmid with the wild type ASS CDNA (left panel, WT) and cloned fibroblast CDNA of citrullinaemia AC (right panel, AC). CDNA was synthesised using primer E16-1 while that from the RNA-negative allele was specifically amplified using the primer pair E7-2 and E16-2. The products were cloned and analysed by DNA sequencing. A C-to-T transition in exon 12 is revealed. The boundary of exons 12 and 13 is indicated. (C) DNA sequence analysis of PCR product amplified from genomic DNA of the control HuH-7 cell line (left panel, WT) and citrullinaemia AC (right panel, AC) using a pair of primers located in exon 12 [E12, 5'-(791)

GCCGTATTGACATCGTGGAG (810)-3'] and intron 12 (I12-2, 5'ACAGTGTCTCAGGGATCTCT-3'). An extra T band appears in the sequence of the genomic DNA of citrullinaemia AC where a normal $\mathrm{C}$ band is found in the HuH-7 cell line. The boundary of exon 12 and intron 12 is indicated. (D) Schematic diagram of cDNA of the wild type allele and the RNA-negative allele of citrullinaemia AC. A C-to-T transition at nucleotide 835 of cDNA of the RNA-negative allele results in the conversion of the CGA arginine codon at residue 279 to a TGA termination codon.

allele was analysed by allele-specific PCR. Hence, there is no indication of nonsense-associated altered splicing (NAS) associated with R279X mutation.

\section{Discussion}

This study shows that a nonsense mutation at codon 279 within exon 12 of the human argininosuccinate synthetase 


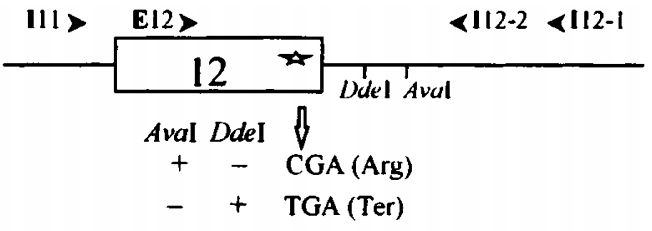

(A) Exon 12-Intron 12

(B) Intron 11-Exon 12-Intron 12
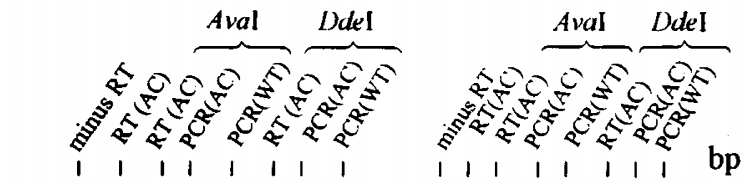

$118-$

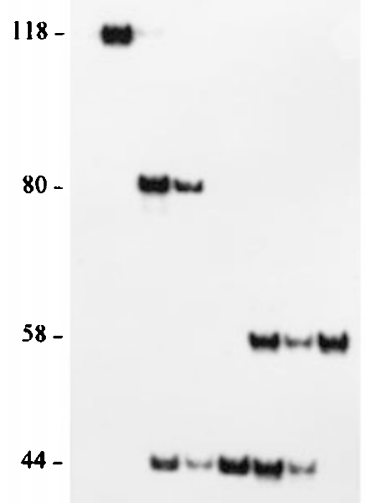

Figure 3 Levels of pre-mRNA containing intron immediately upstream and downstream of the nonsense mutation. (A) Total RNA of citrullinaemia fibroblasts AC was reverse-transcribed using primer 112-1 (5'-CACTTGGCAGGAGGGAAACT-3'). The region containing exon 12-intron 12 sequence was amplified using primers E12 and I12-1, followed by nested PCR using primers E12 and 112-2. The primer E12 was $5^{\prime}$ end-labelled with $\left[\gamma-{ }^{32}\right.$ P $]$ ATP. (B) Total RNA of citrullinaemia fibroblasts AC was reverse-transcribed using primer 112-1.The region containing intron 11-exon 12-intron 12 sequence was amplified using primers 111 (5'-CCGAACCTAATGGACCAGTT-3') and I12-1, followed by nested PCR using primers 111 and $112-2$ with endlabelled primer 111. Lane 'minus $\mathrm{RT}^{\prime}$ ' represents a RT - PCR reaction control in the absence of reverse transcriptase, lane RT(AC) was RT - PCR using total RNA of citrullinaemia fibroblasts $A C$ as template while lanes PCR(AC) and PCR(WT) indicate PCR using genomic DNAs of citrullinaemia $A C$ and normal control as template, respectively. The labelled products were digested with restriction enzyme Aval or Ddel, separated by $8 \%$ polyacrylamide gel electrophoresis and quantified by Phosphorlmager analysis (Molecular Dynamics, USA). Asterisk $(\lesssim)$ in exon 12 marks the position of the C-to-T transition. The expected cutting site of $A v a l$ and Ddel (+, with site; -, without site) in the normal and the mutant alleles and the common cutting site within the amplified region are indicated.

gene is responsible for an RNA-negative phenotype. This is the first nonsense mutation identified in human citrullinaemia. Nonsense mutation-mediated mRNA reduction has been reported in many studies. ${ }^{11-13}$ It can result in degradation of mRNA within cytoplasm or mRNA asso- ciated with nucleus. It can also influence the choice of splice sites resulting in intron retention, exon skipping or activation of cryptic splice site. Our study of the half-life of cytoplasmic mRNA degradation by RT-PCR after actinomycin D treatment do not support the view that cytoplasmic mRNA degradation is the major event responsible for the RNA-negative phenotype in citrullinaemia patient AC (data not shown). Quantification of the steady-state levels of pre-mRNAs from the endogenous gene bearing the nonsense mutation shows no accumulation of either pre-mRNA bearing intron immediately upstream or downstream of the affected exon. In addition, there is no evidence to support exon skipping associated with this mutation. Therefore, the most likely defect responsible for the $\mathrm{R} 279 \mathrm{X}$ mutation-mediated mRNA reduction is an event affecting the abundance of nucleus-associated mRNA. We estimate that mRNA from an allele carrying the R279X mutation is less than $2 \%$ of the normal level. Likewise, the abundance of mRNA in the liver of bovine citrullinaemia caused by a nonsense mutation at codon 86 was estimated to be less than 5\% of normal. ${ }^{15}$ On the other hand, studies in the human triosephosphate isomerase (TPI) gene has shown that the abundance of the mRNA decreases to approximately $20 \%$ of the normal level when nonsense mutations are located within the first three quarters of the reading frame. ${ }^{16}$ In addition, a study of the adenine phosphoribosyltransferase (aprt) gene in $\mathrm{CHO}$ cells has shown a 5-10-fold decrease in steady-state mRNA levels in mutants in exons 1,2 , and 4 but mutations in the $3^{\prime}$-most exon exert little effect. ${ }^{17}$ The human argininosuccinate synthetase gene contains 16 exons with an open reading frame of $1236 \mathrm{nt}$, while R279X mutation is located at nt 835 of exon 12. It is not clear why its mRNA level is low when harbouring the R279X mutation. It is noteworthy that within cytoplasm, mRNAs of the wild type allele and mutant harbouring the R279X mutation were equally stable with a half-life longer than $20 \mathrm{~h}$ (unpublished data). On the other hand, a nuclear post-transcriptional event has been shown to be responsible for ASS mRNA overproduction in a canavanine-resistant variant of a human epithelial cell line involving some labile protein factor(s). ${ }^{10}$ It will be of interest to elucidate how regulation of the decay of the ASS mRNA mediated by the nonsense mutation fits into the regulatory scheme of mRNA overproduction in canavanine-resistant cells since both events associate with nucleus.

\section{Acknowledgments}

We thank Dr K-B Choo for helpful discussion and critical reading of the manuscript, Drs AL Beaudet and WE O'Brian (Baylor College of Medicine) for providing skin fibroblasts of citrullinaemia patients. This work was supported in part by grants NSC 86-2314-B-075-084 and NSC 87-2314-B-075-089 from the National Science Council, and by a grant from Veterans General Hospital, Taipei. 


\section{References}

1 Brusilow SW, Horwich AL: Urea cycle enzyme; In Scriver C, Beaudet A, Sly W, Valee D (eds): Metabolic and molecular bases of inherited disease. McGraw-Hill, New York, 1995, 7th edn, pp $1187-1232$.

2 Su TS, Beaudet AL, O'Brien WE: Abnormal mRNA for argininosuccinate synthetase in citrullinemia. Nature 1983; 301: $533-$ 534.

3 Beaudet AL, O'Brien WE, Bock HGO, Freytag SO, Su TS: The human argininosuccinate synthetase locus and citrullinemia; in Harris H, Hirschhorn K (eds): Advances in Human Genetics. New York, 1986, vol 15, pp 161-196.

4 Su TS, Lin LH: Analysis of a splice acceptor site mutation which produces multiple splicing abnormalities in the human argininosuccinate synthetase locus. J Biol Chem 1990; 265: $19716-19720$.

5 Kobayashi K, Jackson MJ, Tick DB, O'Brien WE, Beaudet AL: Heterogeneity of mutations in argininosuccinate synthetase causing human citrullinemia. J Biol Chem 1990; 265: 1136111367.

6 Kobayashi K, Shaheen N, Terazono H, Saheki T: Mutations in argininosuccinate synthetase mRNA of Japanese patients, causing classical citrullinemia. Am J Hum Genet 1994; 55: $1103-1112$

7 Nakabayashi HK, Taketa K, Miyano K, Yamane T, Sato J: Growth of human hepatoma cell lines with differentiated functions in chemically defined medium. Cancer Res 1982; 42: 3858 - 3863 .

8 Sambrook J, Fritsch EF, Maniatis T: Molecular cloning: a laboratory manual, Cold Spring Harbor, Cold Spring Harbor Press, 1989, 2nd edn.
9 Innis MA, Gelfand DH, Sninsky JJ, White TJ: PCR protocols, a guide to methods and applications. San Diego, Academic Press, 1990.

10 Tsai TF, Su TS: A nuclear post-transcriptional event responsible for overproduction of argininosuccinate synthetase in a canavanine-resistant variant of a human epithelial cell line. Eur J Biochem 1995; 229: 233-238.

11 Maquat LE: Defects in RNA splicing and the consequence of shortened translational reading frames. Am J Hum Genet 1996; 59: $279-286$

12 Hentze MW, Kulozik AE: A perfect message: RNA surveillance and nonsense-mediated decay. Cell 1999; 96: 307-310.

13 Cooper TA, Mattox W: The regulation of splice-site selection, and its role in human disease. Am J Hum Genet 1997; 61: 259266.

14 Liu H-X, Cartegni L, Zhang MQ, Krainer AR: A mechanism for exon skipping caused by nonsense or missense mutations in BRCA1 and other genes. Nature Genet 2001; 27: 55-58.

15 Dennis JA, Healy PJ, Beaudet AL, O'Brien WE: Molecular definition of bovine argininosuccinate synthetase deficiency. Proc Natl Acad Sci USA 1989; 86: 7947 - 7951.

16 Cheng J, Maquat LE: Nonsense codons can reduce the abundance of nuclear mRNA without affecting the abundance of pre-mRNA or the half-life of cytoplasmic mRNA. Mol Cell Biol 1993; 13: $1892-1902$

17 Kessler O, Chasin LA: Effects of nonsense mutations on nuclear and cytoplasmic adenine phosphoribosyltransferase RNA. Mol Cell Biol 1996; 16: 4426-4435. 